\title{
CARACTERIZAÇÃO MORFOLÓGICA E PADRĀO DE FLORESCIMENTO DE TEOSINTOS CULTIVADOS SOB FOTOPERÍODO CURTO E SUAS IMPLICAÇŌES NA ANÁLISE FENÉTICA
}

\author{
Ana Beatriz Ferreira Torreau' \\ William José da Silva ${ }^{2}$
}

\begin{abstract}
RESUMO - O efeito do fotoperíodo curto na morfologia e biologia reprodutiva dos teosintos, Zea mays ssp. mexicana (Schrader) Iltis (raças Central Plateau e Chalco Los Reyes), Z. mays ssp. parviglumis var. huehuetenanguensis (Iltis \& Doebley), Z. luxurians (Durieu \& Ascherson) Bird e Z. diploperennis (Iltis, Doebley \& Guzmán) e do milho mazóide I-8, Z. mays ssp. mays L., foi estudado na Área Experimental da UNICAMP (SP) $22^{\circ} 54^{\prime}$ latitude sul, de fevereiro a julho de 1989. Os representantes da espécie Z. mays ssp. mexicana foram os mais precoces, seguidos do Z. mays ssp. mays, Z. diploperennis, Z. luxurians e Z. mays ssp. parviglumis var. huehuetenanguensis. Pela análise canônica, observou-se que esses acessos de germoplasma estâo distribuídos em 4 diferentes grupos, onde as características: início e fim da antese na panícula terminal e início e fim da antese nas panículas dos ramos laterais foram as principais responsáveis pela discriminação das várias populações de teosinto e do milho. Z. mays ssp. parviglumis var. huehuetenanguensis foi colocada num grupo intermediário entre representantes da Seção Zea e da Seção Luxuriantes. Esses resultados corroboram dados recentes de outros pesquisadores baseados em análises isoenzimáticas, e discordam portanto da classificação de Doebley \& Iltis (1980), baseada na morfologia das inflorescências masculinas e forma da cúpula que reveste o fruto.
\end{abstract}

Palavras-chave: Teosinto, Fotoperíodo, Zea e Luxuriantes.

ABSTRACT - The effect of short daylength on the morphology and reproduction of teosinte, Zea mays ssp. mexicana (Schrader) Iltis (races Central Plateau and Chalco Los Reyes), Z. mays ssp. parviglumis var. huehuetenanguensis (Iltis \& Doebley), Z. luxurians (Durieu \& Ascherson) Bird and Z. diploperennis (Iltis, Doebley \& Guzmán) were studied in comparison with a maize population at the experimental area of UNICAMP (SP), $22^{\circ} 54^{\prime} \mathrm{S}$, on February-July 1989. Daily phenological observations showed that $Z$. mays ssp. mexicana populations were the earliest to flower followed by Z. mays ssp. mays, Z. diploperennis, Z. luxurians and $Z$. mays ssp. parviglumis var. huehuetenanguensis. According to a canonical analysis it was found that these germplasm accessions were distributed in four different

1 - Depto de Genética Cx. P. 6109 UNICAMP, Campinas, SP (Bolsista da Capes/PICD - UFMA)

2 - Dept ${ }^{\circ}$ de Genética e Evolução - UNICAMP 
groups according to the traits begining and ending of flowering of terminal panicles, and begining and ending of flowering of panicles of lateral branches. The population of $Z$. mays ssp. parviglumis var. huehuetenanguensis was placed as a intermediary group between Section Zea and Section Luxuriantes. These findings agree with recent works based on isoenzymatic analysis and disagree with the classification by Doebley \& Iltis (1980) based on morphology of male inflorescence and shape of the cupule.

Key-words: Teosinte, photoperiod, Zea e Luxuriantes.

\section{Introdução}

Teosinto e milho pertencem à tribo Andropogoneae e à família Gramineae. Estudos de biossistemática parecem sugerir que o teosinto, espécie selvagem, seja o provável ancestral do milho (de Wet \& Harlan, 1972; Beadle, 1980):

A discriminação entre o germoplasma selvagem e o domesticado pode ser feita através do estudo de características morfofisiológicas, uma vez que são alteradas freqüentemente ao longo do processo de domesticação. Dentre essas, há grande interesse no estudo da resposta da planta à fotossensibilidade, uma vez que tem sido detectado por vários autores (Wilkes, 1967; Beadle, 1980; Fridman, 1987) que o teosinto parece ser mais sensível ao fotoperíodo que o milho.

O fato de que a resposta da planta ao fotoperíodo parece estar estreitamente ligada à sua arquitetura (Fridman, 1987), estimulou a realização do presente trabalho para melhor conhecer o efeito de dias curtos em características morfofisiológicas durante a reprodução.

\section{Material e métodos}

As análises canônica e fenética dos acessos de germoplasma (Figura $1 \mathrm{e}$ 2) foram feitas com base nos dados de fenologia reprodutiva e de morfofisiologia de cada população. Os primeiros correspondem ao número de dias para o início e fim da antese nas panículas terminais e nas panículas dos ramos laterais (teosintos), bem como o período de saída dos estigmas numa amostra de 100 plantas tiradas ao acaso de cada população. Os dados morfofisiológicos obtidos a partir de 20 plantas, também tomadas ao acaso foram: número de perfilhos, número de nós, número de frutos totais, comprimento e número de espiguetas em $5 \mathrm{~cm}$ da porção média da ráquis central da panícula terminal, altura da planta, comprimento e largura da folha média, peso seco da parte aérea e peso dos frutos. Esses conjuntos de dados permitiram a discriminação e avaliação do grau de similaridade dos acessos de germoplasmas estudados. 


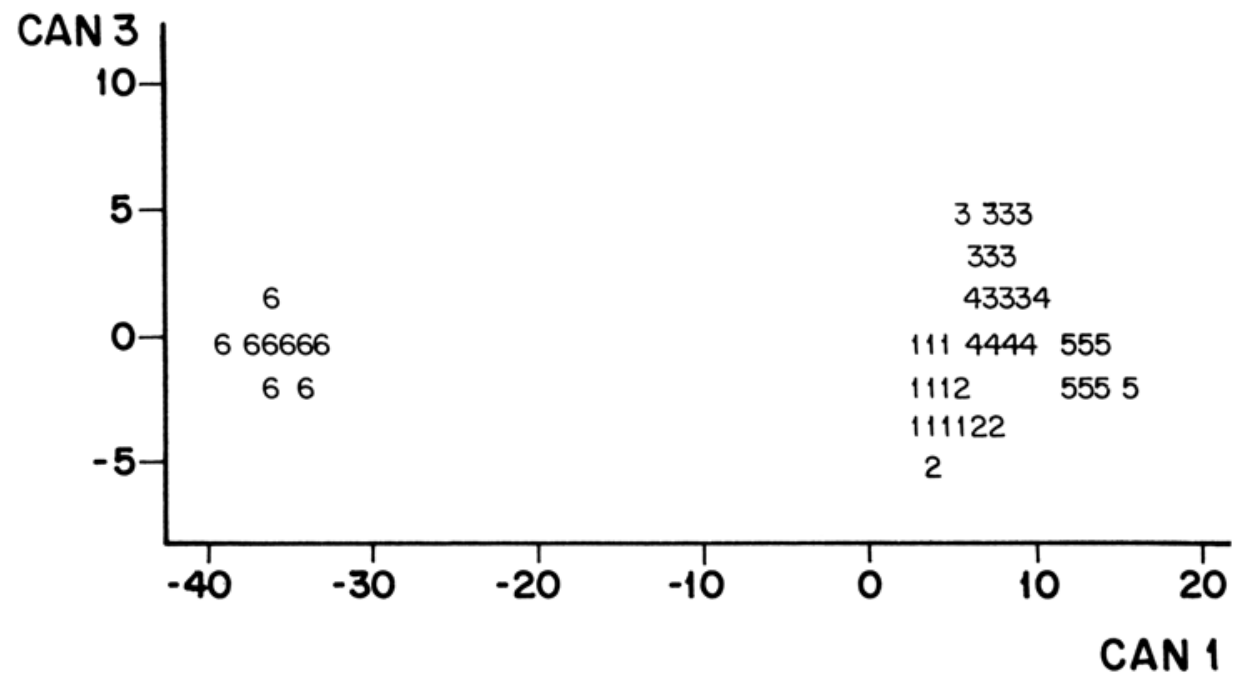

Figura 1: Gráfico de correlação canônica das variáveis morfológicas tomadas nas populações de milho e teosinte. Eixo das abscissas-variável canônica 1 e eixo das ordenadas variável canônica 3 .

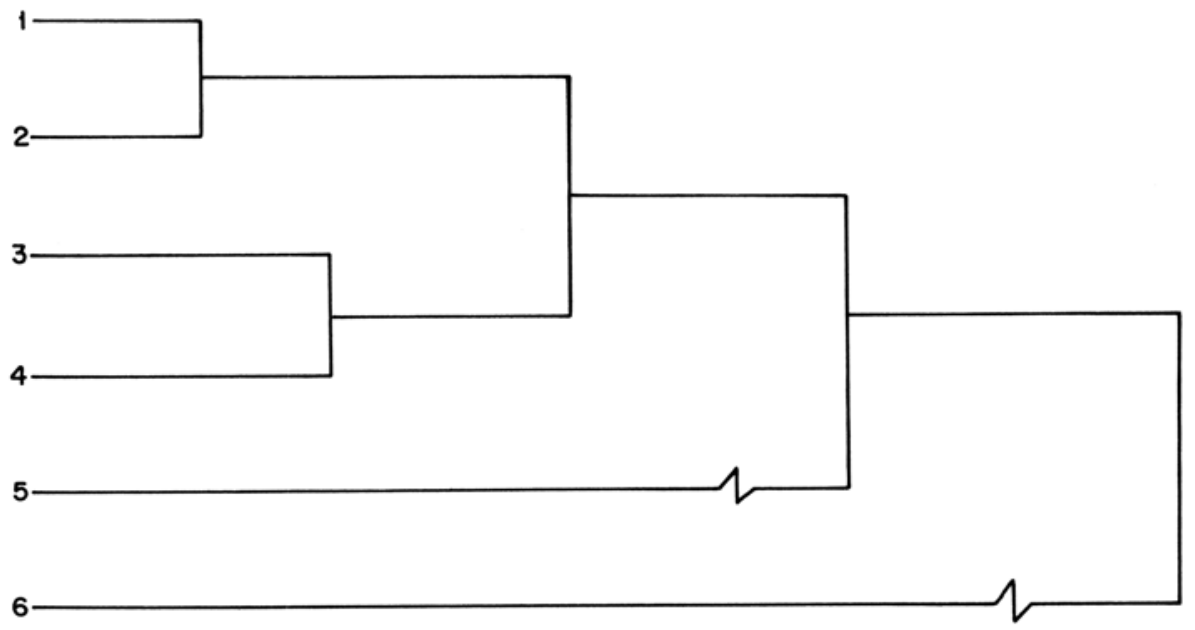

$\begin{array}{lllll}23.46 & 26.97 & 50.42 & 203.53 & 1943.20\end{array}$

Figura 2: Fenograma baseado nas distâncias de Mahalanobis das populações de milho e teosinte. 1 - Zea mays ssp. mexicana (Central Plateau); 2 - Z. mays ssp. mexicana (Los Reyes); $3-Z$. diploperennis; $4-Z$. luxurians; $5-Z$. mays ssp. parviglumis var. huehuetenanguensis; 6 - Z. mays ssp mays. 


\section{Resultados}

Com base na análise canônica, verificou-se que a variável CAN1 apresentou maior significância como um fator discriminante na proporção de 0.9069. O início e fim da antese nas panículas terminais e nas panículas dos ramos laterais foram as características que apresentaram os maiores coeficientes canônicos, indicando serem as mesmas as principais responsáveis pela separação das populações. A Figura 1 demonstra que em relação à variável CAN1, houve a discriminação dos materiais em dois grupos principais: o da população de milho (6) com valores negativos e o dos teosintos $(1,2,3,4$ e 5) com valores positivos. Pode-se verificar ainda um agrupamento seqüencial dos teosintos. Um primeiro grupo formado pelos teosintos anuais da espécie $Z$. mays ssp. mexicana, raças Central Plateau (1) e Los Reyes (2). Um segundo grupo, com os teosintos da seção Luxuriantes, a espécie perene $Z$. diploperennis (3) e a anual, $Z$. luxurians (4) e isoladamente o terceiro grupo de teosinto anual, $Z$. mays ssp. parviglumis var. huehuetenanguensis (5), pertencente à seção Zea. Esse último parece apresentar diferenças significativas em relação aos outros acessos de germoplasma das seções Luxuriantes e Zea.

As distâncias de Mahalanobis (Tabela 1) serviram à análise fenética dos materiais, e a partir das mesmas foi construído o fenograma da figura 2. Nota-se claramente, que os teosintos $Z$. mays ssp. mexicana e os componentes da seção Luxuriantes, $Z$. luxurians e $Z$. diploperennis, agruparam-se em dois diferentes "clusters", dois a dois, comprovando a forte similaridade entre os mesmos. Já Z. mays ssp. parviglumis var. huehuetenanguensis tomou uma posição intermediária em relação ao milho e aos demais teosintos, como demonstrado na análise canônica, sugerindo que a mesma não está bem situada como componente da seção Zea.

Tabela 1 - Distância de Mahalanobis estimadas a partir de 10 características estudadas nas populações: $Z$. mays ssp. mexicana Central Plateau (1); $Z$. mays ssp. mexicana Chalco Los Reyes (2); Z. diploperennis (3); Z. Iuxurians (4); $Z$. mays ssp. parviglumis var. huehuetenanguensis (5) e $Z$. mays ssp. mays (6).

\begin{tabular}{lrrrrrr}
\hline Germoplasmas & \multicolumn{1}{c}{1} & 2 & 3 & 4 & 5 & 6 \\
1 & 0.000 & & & & & \\
2 & 23.464 & 0.000 & & & & \\
3 & 52.227 & 45.894 & 0.000 & & & \\
4 & 65.276 & 38.276 & 26.967 & 0.000 & & \\
5 & 314.293 & 194.044 & 185.959 & 119.830 & 0.000 & \\
6 & 1636.000 & 1747.000 & 1907.000 & 1946.000 & 2480.000 & 0.000 \\
\hline
\end{tabular}


As observações aqui coletadas confirmam resultados obtidos por Doebley et al. (1984), Mastenbroek et al. (1981) e Mastenbroek \& de Wet (1983). Esses resultados apontam a necessidade da realização de mais estudos sobre a sistemática do gênero Zea com base em outros caracteres, além dos morfológicos explorados por Doebley \& Iltis (1980), para elucidar definitivamente a distribuição das espécies nas seções Zea e Luxuriantes.

\section{Agradecimentos}

Ao prof. Dr. Sérgio dos Reis, Depto de Parasitologia (IB - UNICAMP) pelo auxílio no processamento dos dados.

\section{Referências bibliográficas}

BEADLE, G.W. 1980. The ancestry of corn. Scientific American, 242: 96-103.

WET, J.M.J. \& J.R. HARLAN, 1972. Origin of maize: The tripartite hypothesis. Euphytica, 21: 271-279.

DOEBLEY, J.F., M.M. GOODMAN \& C.W. STUBER. 1984. Isoenzimatic variation in Zea (Gramineae). Syst.Bot. 9(2): 203-218.

DOEBLEY, J.F. \& H.H. ILTIS, 1980. Taxonomy of Zea (Gramineae). I. A subgeneric classification with key to taxa. Am.J. Bot.67(6): 982-993.

FRIDMAN, J. 1987. Fotoperiodismo e competiçâo de grão de pólen como mecanismos de isolamento genético entre milho e teosinte. Dissertação de Mestrado. Unicamp. Campinas, São Paulo, 91p.

MASTENBROEK, I \& J.M.J. de WET, 1983. Chromosome C-banding of Zea mays and its closest relatives. Canadian Journal of Genetics and Cytology 25: 203-209.

MASTENBROEK, I., C.E. COHEN \& J.M.J. de WET, 1981. Seed protein and seedling isozyme patterns of Zea mays and its closest relatives. Biochemistry Systematics and Ecology, 9(2/3): 179-183.

WILKES, H.G. 1967. Teosinte: the closest relative of maize. The Bussey Inst. of Harvard University, Cambridge, Mass. 159p. 